\title{
Susceptibility Profile of Candida albicans Biofilms on Polyvinyl Chloride Endotracheal Tube to Antifungal Azoles
}

\author{
Derick Erl P. Sumalapao ${ }^{1,2 *}$ (D), Frederico Martin D. Alegre ${ }^{2,3}$ (D), Pauline Bridgette \\ D. Salazar ${ }^{2,3}\left(\mathbb{D}\right.$, Nelson R. Villarante ${ }^{4}$, Isidro C. Sia ${ }^{5,6}$ (D) and Nina G. Gloriani ${ }^{7}$
}

${ }^{1}$ Department of Epidemiology and Biostatistics, College of Public Health, University of the Philippines Manila, Manila, Philippines. ${ }^{2}$ Biology Department, College of Science, De La Salle University, Manila, Philippines.

${ }^{3}$ College of Medicine, De La Salle Medical and Health Sciences Institute, Cavite, Philippines. ${ }^{4}$ Department of Physical Sciences and Mathematics, College of Arts and Sciences, University of the Philippines Manila, Manila, Philippines. ${ }^{5}$ Department of Pharmacology and Toxicology, College of Medicine, University of the Philippines Manila, Manila, Philippines. ${ }^{6}$ Integrative Medicine for Alternative Healthcare Systems Philippines, Inc., Quezon City, Philippines. ${ }^{7}$ Department of Medical Microbiology, College of Public Health, University of the Philippines Manila, Manila, Philippines.

\begin{abstract}
One of the most prevalent causative organisms in human fungal infections is Candida albicans. The extensive utility of medical devices such as endotracheal tube has resulted in the increased cases of fungal infections. The present study visualized Candida albicans biofilm on polyvinyl chloride endotracheal tube and described the susceptibility profile of the biofilm to antifungal azoles. Biofilm was examined using light and fluorescence microscopy. The susceptibility profile of planktonic and sessile cells with posaconazole, miconazole, voriconazole, and fluconazole was assessed employing the standard disc diffusion method. Planktonic $C$. albicans was susceptible to the identified antifungal azoles, whereas the sessile cells had varying susceptibility profiles. The biofilms remained susceptible to voriconazole and fluconazole, susceptible-dose dependent to posaconazole, and resistant to miconazole. Findings of the present study can provide relevant information on the resistance mechanism of fungal biofilms with azoles and in the design of new pharmacologic interventions intended as treatment of mycoses.
\end{abstract}

Keywords: Fluconazole, fungal infections, miconazole, polymer-based material, posaconazole, standard disc diffusion method, voriconazole

*Correspondence: dpsumalapao1@up.edu.ph

(Received: February 19, 2020; accepted: March 21, 2020)

Citation: Sumalapao DEP, Alegre FMD, Salazar PBD, et al. Susceptibility Profile of Candida albicans Biofilms on Polyvinyl Chloride Endotracheal Tube to Antifungal Azoles. J Pure Appl Microbiol. 2020;14(2):1143-1148. doi: 10.22207/JPAM.14.2.09

C The Author(s) 2020. Open Access. This article is distributed under the terms of the Creative Commons Attribution 4.0 International License which permits unrestricted use, sharing, distribution, and reproduction in any medium, provided you give appropriate credit to the original author(s) and the source, provide a link to the Creative Commons license, and indicate if changes were made. 


\section{INTRODUCTION}

Medical devices are utilized in clinical, therapeutic, and diagnostic procedures (Stoica et al. 2017). Endotracheal tube is one of the widely used devices in clinical practice particularly in ventilator-associated pneumonia, subglottic stenosis, and bronchopulmonary dysplasia (Gibbs \& Holzman 2012). Polyvinyl chloride is one of the most common materials used in making endotracheal tube due to its capability to contain large volume and yet maintain a low pressure which controls the full inflation of the cuff preventing damage on tracheal wall (Mariyaselvam et al. 2017). The utility of medical devices was linked with device-associated infections with Candida as the most common fungal genus found to colonize these medical devices (Desai et al. 2014). There has been a lot of focus on the growth patterns of Candida in the recent years as these caused quite a number of infections in the body in their pathogenic phase (Tsui et al. 2016). They grow in a mucosal environment allowing them to thrive on medical devices such as the endotracheal tube (Tsui et al. 2016). The genus Candida, the fourth most common nosocomial bloodstream pathogen, was reported to cause almost $90 \%$ of invasive fungal infections with $45 \%$ mortality rate (Spampinato \& Leonardi 2013).

Furthermore, Nobile and Johnson (2015) reported in their study that National Institutes of Health identified biofilm formation of $C$. albicans to be the cause of $80 \%$ of all microbial infections, ranging from superficial mucosa to more serious infections, with high mortality rates. In the treatment of these fungal infections, azoles are frequently used because of their availability for oral administration, economical price, and less toxicity (Whaley et al. 2016). Hence, the present study assessed the susceptibility of $C$. albicans biofilms on polyvinyl chloride endotracheal tube (PVC-ETT) to antifungal azoles using standard disc diffusion method. Information on model fungal biofilms with emphasis on susceptibility profile can provide possible explanations in the elucidation of resistance patterns of fungal biofilms with the currently available antifungal drugs designed primarily in the treatment of local and systemic mycoses. New features of these fungal biofilms can provide additional information in the subsequent synthesis of pharmacologic interventions intended for biofilm-associated diseases. In addition, new insights will influence other disciplines such as biomedical engineering in the manufacture of medical devices to minimize occurrence of biofilmrelated infections.

\section{MATERIALS \& METHODS \\ Organism and Inoculum}

In this investigation, C. albicans was subcultured on Sabouraud dextrose agar (SDA) and incubated at $37^{\circ} \mathrm{C}$ for $48 \mathrm{~h}$. The inoculum was prepared using an existing protocol (Andes et al. 2004) with minor adjustments. Specifically, three colonies were added in $10 \mathrm{~mL}$ sterile distilled water. Using serial dilution, viable fungal counts were adjusted to $7.46( \pm 0.07) \log _{10} \mathrm{CFU} / \mathrm{mL}$.

\section{Biofilm Formation}

To generate fungal biofilms, an existing protocol (Chandra et al. 2001) was followed with slight modifications. Disks with the dimensions of $0.2 \mathrm{~cm} \times 0.8 \mathrm{~cm}$ were carefully cut from the PVCETT (Sacett, Portex) and subsequently sterilized. The dried disks were individually placed in 96-well culture plates and $100 \mu \mathrm{L}$ of the prepared inoculum with $200 \mu \mathrm{L} 50 \mathrm{mM}$ glucose were introduced in each disk. The plates were then incubated at $37^{\circ} \mathrm{C}$ for a period of $72 \mathrm{~h}$. For the microscopic studies of biofilms, nonadherent cells were removed by washing the disks with $300 \mu \mathrm{L}$ of $0.15 \mathrm{M}$ phosphate buffer solution (PBS). The disks were then examined using a light microscope. For the fluorescence microscopy, the disks were stained with $2 \mathrm{~mL}$ dye (Sypro ruby) for $15 \mathrm{~min}$ prior to microscopic examination.

\section{Antifungal Susceptibility Testing}

Following biofilm formation, standard disc diffusion method was employed to determine zone of inhibition of fluconazole, miconazole, posaconazole, and voriconazole. Standard inoculum was adjusted to $10^{7}$ cells $/ \mathrm{mL}$. After biofilm formation, disks were washed with 0.15 M PBS $(300 \mu \mathrm{L})$. Varied number of strips were transferred to a test tube containing $2 \mathrm{~mL}$ of sterile distilled water until similar turbidity was obtained with the established standard test tube and confirmed by standard plating procedure. Once vortexed, a sterile cotton swab was dipped in the suspension and swabbed over Mueller-Hinton agar (MHA) plate. With a pair of sterile forceps, each antifungal drug was dispensed on the surface of 
the agar. The plates were then incubated at $37^{\circ} \mathrm{C}$ for $24 \mathrm{~h}$ and subsequently the zone of inhibition was measured. All experiments were prepared in triplicate per monitoring time following biofilm formation.

\section{Data and Statistical Analysis}

Evaluation of the susceptibility of $C$. albicans biofilms was based on Clinical and Laboratory Standards Institute (CLSI, 2017) breakpoints for each drug and Susceptibility Testing for Yeasts (STY, 2011) guidelines. Zones of inhibition were reported as mean \pm standard deviation. Means were compared (analysis of variance and Bonferroni test) at $5 \%$ significance level using STATA.

\section{RESULTS}

Candida albicans adhered on PVC-ETT and subsequently formed biofilm (Figs. 1-2). The complexity of the aggregation increased as the biofilm forms with time. Representative images for the susceptibility testing of $C$. albicans biofilms on PVC-ETT to antifungal azoles are presented in Fig. 3. These $C$. albicans biofilms showed varying
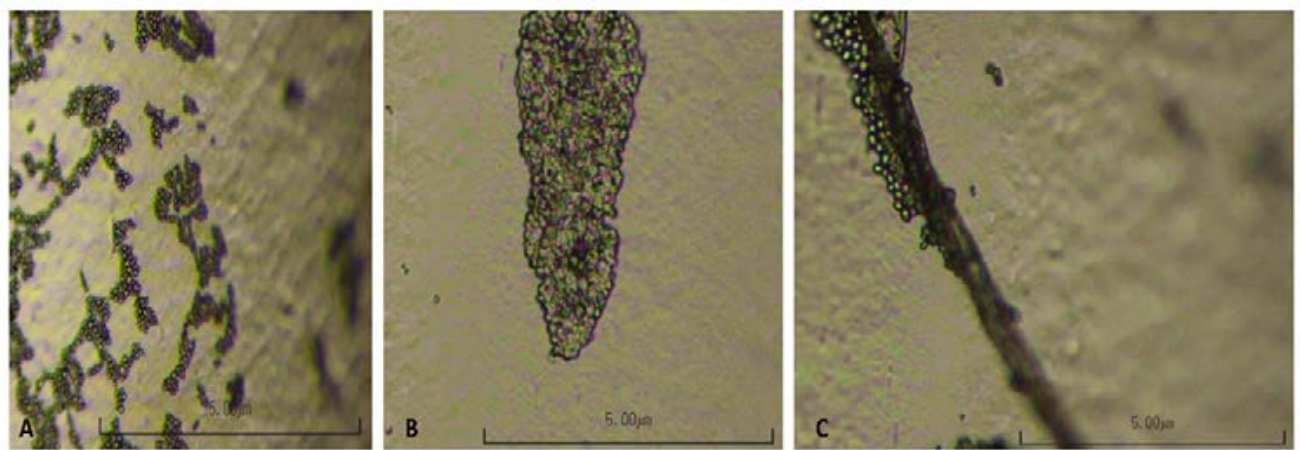

Fig. 1. Candida albicans biofilms on PVC-ETT at (A) 24 h, (B) 48 h, and (C) 72 h under light microscope at high power objective.
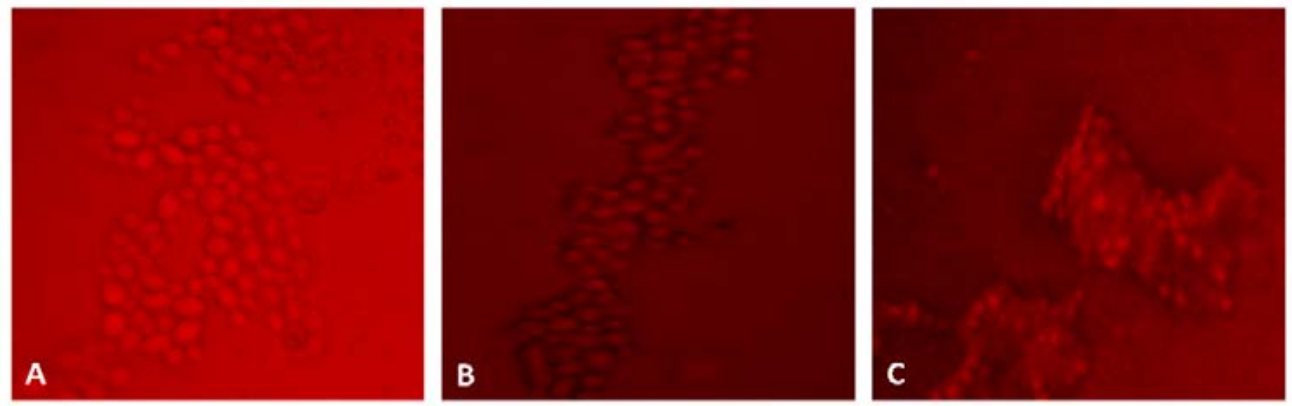

Fig. 2. Visualization of Candida albicans biofilms using fluorescence microscopy (X40 magnification). (A) 24-h, (B) 48-h, and (C) 72-h biofilms.

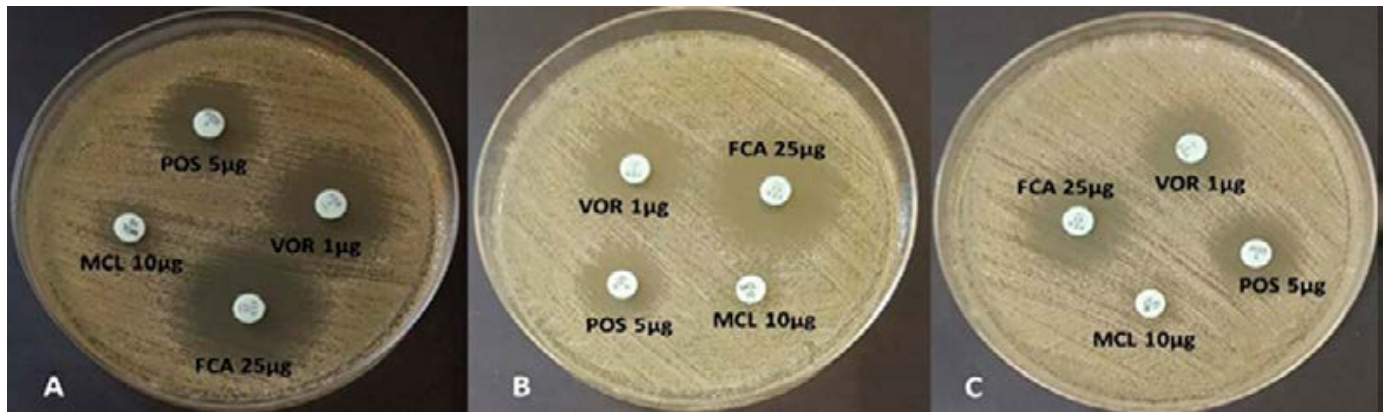

Fig. 3. Susceptibility pattern of Candida albicans biofilms on PVC-ETT against azoles: (A) 24-h, (B) 48-h, (C) 72-h; FCA: fluconazole, MCL: miconazole, POS: posaconazole, VO: voriconazole. 
susceptibility patterns to the antifungal azoles (Table 1). The planktonic cells were susceptible to the antifungal azoles. In the serially grown biofilms, the sessile cells were resistant to miconazole. A change in the susceptibility profile of the sessile cells was observed with posaconazole, from susceptible 48 -h biofilm to susceptible-dose dependent 72-h biofilm. However, C. albicans biofilms remained susceptible to fluconazole and voriconazole.
For fluconazole, the zones of inhibition of the planktonic cells and the biofilms at 24 to $48 \mathrm{~h}$ significantly differed from that of the $72-\mathrm{h}$ biofilm. Significant differences between the zone of inhibition of planktonic cells and the $C$. albicans biofilms with miconazole and posaconazole were also observed. Finally, susceptibility pattern of 24-h and 48-h biofilms with voriconazole was found to be significantly different between the planktonic cells and the 72-h biofilm.

Table 1. Antifungal susceptibility of Candida albicans planktonic cells and biofilms on PVC-ETT.

\begin{tabular}{|c|c|c|c|c|}
\hline \multirow[t]{2}{*}{ Azoles } & \multicolumn{4}{|c|}{ Zone of inhibition (mean \pm standard deviation, $\mathrm{mm}$ ) } \\
\hline & Planktonic cells & 24-h biofilm & 48-h biofilm & 72-h biofilm \\
\hline Voriconazole $(1 \mu \mathrm{g})$ & $23.67 \pm 0.58^{\mathrm{a}}$ & $22.00 \pm 0.00^{b}$ & $21.33 \pm 0.58^{b}$ & $18.33 \pm 0.58^{c}$ \\
\hline Miconazole $(10 \mu \mathrm{g})$ & $21.33 \pm 0.58^{a}$ & $10.67 \pm 1.15^{b}$ & $10.00 \pm 1.00^{b}$ & $9.33 \pm 0.58^{b}$ \\
\hline Fluconazole $(25 \mu \mathrm{g})$ & $28.33 \pm 0.58^{\mathrm{a}}$ & $26.67 \pm 0.58^{a}$ & $26.33 \pm 0.58^{a}$ & $21.00 \pm 1.00^{\mathrm{b}}$ \\
\hline Posaconazole $(5 \mu \mathrm{g})$ & $24.00 \pm 1.00^{\mathrm{a}}$ & $18.00 \pm 1.00^{\mathrm{b}}$ & $18.00 \pm 1.00^{\mathrm{b}}$ & $16.67 \pm 1.53^{b}$ \\
\hline
\end{tabular}

In a given row, means with identical superscripts are not significant at $5 \%$

\section{DISCUSSION}

Biofilms are surface-associated communities that are capable of colonizing the host's tissue or medical devices inside the body (Desai et al. 2014). An increase in the usage of medical devices among immunocompromised patients was found to be parallel with devicerelated biofilm infections caused by $C$. albicans (Shinde et al. 2012). Recurring infections and death resulted from these Candida biofilms (Pandolfi et al. 2019). Such clinical problem is further aggravated by its resistance to antifungal drugs. Azoles, the largest family of antifungal drugs, include imidazoles (e.g. miconazole) and triazoles (e.g. fluconazole, posaconazole, and voriconazole). In this study, planktonic cells and biofilms on PVC-ETT exhibited consistent susceptibility profile to fluconazole and voriconazole. Fluconazole, a broad spectrum antifungal drug, remains the first choice in the treatment of fungal infections because of its high efficacy, safety profile, and bioavailability (Liu et al. 2015). However, 14.1\% fluconazole resistance to $C$. albicans was reported in 2014 due to repeated use of the drug (Liu et al. 2015). Fluconazole targets $C$. albicans by inhibiting lanosterol demethylase in the synthesis of ergosterol and such inhibition promotes toxic accumulation of methylated sterols in fungal cell which eventually leads to cell death (Berkow \& Lockhart 2017). Voriconazole and posaconazole are both broad-spectrum second-generation triazoles that are structurally similar to fluconazole and itraconazole, respectively, but are reserved for invasive infections (Ashbee et al. 2014). Voriconazole is also an active triazole derived from fluconazole with enhanced antifungal spectrum (Saravolatz et al. 2003). Furthermore, voriconazole and other second-generation azoles were also reported to be fungicidal for some filamentous organisms (Saravolatz et al. 2003).

Susceptibility pattern of $C$. albicans biofilms on PVC-ETT with posaconazole also changed from susceptible to resistant, although no significant difference was reported. Posaconazole is a broad-spectrum triazole used to treat infections caused by Candida, Aspergillus, and other opportunistic and dimorphic fungi encountered clinically (Gonzalez et al. 2007). However, increased resistance with posaconazole is presumably caused by a sixth mutation in ERG11, which functions to disrupt the binding segments of the drugs, elevating the level of posaconazole resistance (Li et al. 2015). Moreover, similar susceptibility pattern was also observed for miconazole, although only 
the planktonic cells were susceptible and the rest of the serially grown biofilms were found to be resistant. Miconazole was the first azole identified to be effective against systemic infections (Loefler \& Stevens 2003). As was described for triazoles, miconazole also functions to affect membrane asymmetry and fluidity, and this altered plasma membrane composition may have contributed to the observed resistance to miconazole (Loefller \& Stevens 2003).

\section{CONCLUSION}

Biofilms on PVC-ETT exhibited varying susceptibility patterns with antifungal azoles. Candida albicans biofilms were susceptible to fluconazole, posaconazole, and voriconazole, but resistant to miconazole. The findings of the present study can provide additional information in the elucidation of resistance mechanism of the fungal biofilms with the existing antifungal drugs. Extensive study on the adherence of $C$. albicans on PVC-ETT would provide additional relevant information in the design and manufacture of biomedical devices potentially inhibiting biofilm formation and preventing development of possible fungal biofilm-associated diseases.

\section{ACKNOWLEDGMENTS}

The organism used in this study was kindly provided by the Department of Medical Microbiology, College of Public Health, University of the Philippines Manila. Light and fluorescence microscopies were performed in the laboratories of De La Salle University, Manila, Philippines.

\section{CONFLICT OF INTEREST}

The authors declare that there is no conflict of interest.

\section{AUTHORS' CONTRIBUTION}

All authors listed have made a substantial, direct, and intellectual contribution to the work, and approved it for publication.

\section{FUNDING}

None.

\section{DATA AVAILABILITY}

All data sets analyzed in the study are included in the manuscript and presented as tables and figures.

\section{ETHICS STATEMENT}

The study protocol was approved by the University of the Philippines Manila Research Ethics Board (UPMREB 2018-486-01). The biosafety clearance was granted by the University of the Philippines Manila Institutional Biosafety and Biosecurity Committee (UPMIBBC 2018-018).

\section{REFERENCES}

1. Andes D, Nett J, Oschel P, et al. Development and characterization of an in vivo central venous catheter Candida albicans biofilm model. Infect Immun. 2004;72:6023-6031. https://doi.org/10.1128/ IAI.72.10.6023-6031.2004

2. Ashbee HR, Barnes RA, Johnson EM, et al. Therapeutic drug monitoring (TDM) of antifungal agents: guidelines from the British Society for Medical Mycology. J Antimicrob Chemother. 2014;69:1162-1176. https:// doi.org/10.1093/jac/dkt508

3. Berkow EL, Lockhart SR. Fluconazole resistance in Candida species: a current perspective. Infection and Drug Resistance. 2017;10:237-245. https://doi. org/10.2147/IDR.S118892

4. Chandra J, Kuhn DM, Mukherjee PK, Hoyer LL. Biofilm formation by the fungal pathogen Candida albicans: development, structure, and drug resistance. Journal of Bacteriology. 2001;183:5385-5394. https://doi. org/10.1128/JB.183.18.5385-5394.2001

5. Clinical and Laboratory Standards Institute. CLSI document M60, Performance standards for antifungal susceptibility testing of yeasts, $1^{\text {st }}$ edition. 2017; Wayne, PA 19087 USA.

6. Desai JV, Mitchell AP, Andes DR. Fungal biofilms, drug resistance, and recurrent infection. 2014; Cold Spring Harbor Perspectives in Medicine, 4. https:// doi.org/10.1101/cshperspect.a019729

7. Gibbs K, Holzman IR. Endotracheal tube: friend or foe? Bacteria, the endotracheal tube, and the impact of colonization and infection. Seminars in Perinatology. 2012;36:454-461. https://doi. org/10.1053/j.semperi.2012.06.008

8. Gonzalez GM, Robledo E, Saldivar D, et al. Therapeutic efficacy of posaconazole against isolates of Candida albicans with different susceptibilities to fluconazole in a vaginal model. Medical Mycology. 2007;45:221-224. https://doi.org/10.1080/13693780601164298

9. Li P, Seneviratne CJ, Alpi E, Vizcaino JA, Jin L. Delicate metabolic control and coordinated stress response critically determine antifungal tolerance of Candida albicans biofilm persisters. Antimicrob Agents Chemother. 2015;59:6101-6112. https://doi. org/10.1128/AAC.00543-15

10. Liu J, Shi C, Wang Y, Li W, et al. Mechanisms of azole resistance in Candida albicans clinical isolates from Shanghai, China. Research in Microbiology. 
2015;166:153-161. https://doi.org/10.1016/j. resmic.2015.02.009

11. Loefller J, Stevens DA. Antifungal drug resistance. Clin Infect Dis. 2003;36:S31-S41. https://doi. org $/ 10.1086 / 344658$

12. Mariyaselvam MZ, Marsh LL, Bamford S, et al. Endotracheal tubes and fluid aspiration: an in vitro evaluation of new cuff technologies. $B M C$ Anesthesiology. 2017;17:1-9. https://doi.org/10.1186/ s12871-017-0328-0

13. Nobile CJ, Johnson AD. Candida albicans biofilms and human disease. Annu Rev Microbiol. 2015;69:71-92. https://doi.org/10.1146/annurevmicro-091014-104330

14. Pandolfi F, D’Acierno F, Bortolami M, et al. Searching for new agents active against Candida albicans biofilm: a series of indole derivatives, design, synthesis and biological evaluation. Eur J Med Chem. 2019;165:93106. https://doi.org/10.1016/j.ejmech.2019.01.012

15. Saravolatz LD, Johnson LB, Kauffman CA. Voriconazole: a new triazole antifungal agent. Clin Infect Dis. 2003;36:630-637. https://doi.org/10.1086/367933

16. Shinde RB, Raut JS, Karuppayil MS. Biofilm formation by Candida albicans on various prosthetic materials and its fluconazole sensitivity: a kinetic study. Mycoscience. 2012;53:220-226. https://doi.org/10.1007/S10267011-0155-Y

17. Spampinato C, Leonardi D. Candida infections, causes, targets, and resistance mechanisms: traditional and alternative antifungal agents. Biomed Research International. 2013:1-13. https://doi. org/10.1155/2013/204237

18. Stoica P, Chifiriuc MC, Rapa M, Lazar V. Overview of biofilm-related problems in medical devices. Biofilms and Implantable Medical Device, 2017, 3-23. https:// doi.org/10.1016/B978-0-08-100382-4.00001-0

19. Susceptibility Testing for Yeasts. Agar diffusion method with neo-sensitabs. 2011; Retrieved from www.rosco. dk/gfx/pdf/yeasts.pdf (accessed 22 July 2018).

20. Tsui C, Kong EF, Jabra-Rizk MA. Pathogenesis of Candida albicans biofilm. Pathogens and Disease. 2016;74:ftw018. https://doi.org/10.1093/femspd/ ftw018

21. Whaley SG, Berkow EL, Rybak JM, et al. Azole antifungal resistance in Candida albicans and emerging non-albicans Candida species. Front Microbiol. 2016;7:2173. https://doi.org/10.3389/ fmicb.2016.02173 